[Bailey, C., Hall, W., Millard, D. E. \& Weal, M. J. 2002. Towards Open Adaptive Hypermedia. In Proceedings of the Second International Conference on Adaptive Hypermedia and Adaptive Web Based Systems, Malaga, Spain. 2002. LNCS 2347, Springer, pp. 36-46. (C) Springer-Verlag]

\title{
Towards Open Adaptive Hypermedia
}

\author{
Christopher Bailey, Wendy Hall, David E. Millard, Mark J. Weal \\ IAM Group, ECS, University of Southampton, UK \\ E-mail: \{cpb99r,wh, dem,mjw\}@ecs.soton.ac.uk
}

\begin{abstract}
Research at the University of Southampton has extended generalised Open Hypermedia $(\mathrm{OH})$ models to include concepts of context and behaviour, both traditionally very important to the Adaptive Hypermedia (AH) domain. In this paper we re-evaluate Brusilovsky's pragmatic taxonomy of $\mathrm{AH}$ techniques from a structural perspective. A variety of $\mathrm{OH}$ structures are discussed that can be used to implement the techniques found in the taxonomy. By identifying common structures we gain a new perspective on the relationship between different $\mathrm{AH}$ techniques.
\end{abstract}

Keywords. Open Hypermedia, Adaptive Techniques, Hypermedia Structure, FOHM, taxonomy

\section{Introduction}

In the late 1980's, the hypermedia research community developed, amongst others, two separate research threads; one focusing on Open Hypermedia systems $(\mathrm{OH})$ and one on Adaptive Hypermedia systems (AH). The AH community arose partly from the extensive work that had already been conducted into artificial intelligence and partly from Intelligent Tutoring Systems (ITS). AH researchers are primarily concerned with using pre-existing methods and techniques found in the fields of AI, ITS and User Modelling, and extending, combining and merging these ideas to create complete systems that understand and aid the user in knowledge acquisition.

ITS promoted the development of educational server-side adaptive web-based systems such as MANIC [19], INTERBOOK [5] and more recently AHA [8]. Other such server-side systems index web sites [18] or provide personalized interfaces to large hypermedia systems [9].

AH has also seen the development of client-side adaptive systems that follow users as they browse the WWW. Examples of these systems include WebMate [6], Letizia [14] and LiveInfo [15].

A second thread of hypermedia research focused on the Open Hypermedia $(\mathrm{OH})$ field. Open Hypermedia Systems (OHSs) such as Microcosm [10], Chimera [1] and DHM [11] separate links from documents, allowing hyperstructure to be processed separately from the media it relates to. 
In recent years the $\mathrm{OH}$ community have tackled the issue of interoperability between different OHSs, in particular the development of the Open Hypermedia Protocol (OHP) [7]. The scope of the OHP project evolved into an attempt to create a reference model and architecture for Open Hypermedia Systems in general.

This change has focused the $\mathrm{OH}$ community on the fundamental structures that such systems deal with, which has resulted in the promotion of structure to a firstclass status and the consideration of how context might affect that structure. In particular the development of the Fundamental Open Hypermedia Model (FOHM) [17] deals directly with issues of context, and behaviour that can modify context.

The most recent OHS developed at Southampton, Auld Linky [16], is a contextual structure server that stores and serves structures expressed in FOHM.

\subsection{Motivation}

Our involvement in $\mathrm{OH}$ began with the Microcosm system in 1989 [12]. Although it was not designed as an AH system, Microcosm did provide a framework for building adaptive hypermedia systems. We first reported the use of Microcosm to develop an educational adaptive hypermedia application in [13]. More recently we have developed an adaptive hypermedia system that uses a collection of agents to process the contents of pages visited by the user and augment the current page with contextual links to related information [2].

It is our early experiments with using Linky to support $\mathrm{AH}$ [16] that have led us to the observation that many of the current $\mathrm{AH}$ techniques can be described and supported with a simple set of contextual structures.

The purpose of this paper is to produce an alternative view of the AH domain from a contextually aware $\mathrm{OH}$ perspective, to this end we will be using Brusilovsky's taxonomy of adaptive techniques [4]. We will show that many of the techniques in the taxonomy can be implemented with a small key group of hypermedia structures. This structural perspective is used to analyse and critique the taxonomy and inform the continued development of our contextual link server.

\section{Techniques of Adaptive Hypermedia}

Fig. 1 shows Brusilovsky's taxonomy [4], updated from [3]. This diagram will be used as a basis for a structural comparison of $\mathrm{OH}$ and $\mathrm{AH}$ techniques.

The taxonomy focuses on the interface and user interaction, and has been divided into two distinct areas: 'Adaptive presentation' and 'Adaptive navigation support'.

Adaptive navigation support focuses on aspects of navigational hyperlinks such as generation, appearance, spatial placement and functionality.

Adaptive presentation systems rely on information chunks (or fragments) that can be processed and rendered in a variety of ways depending on the user preferences. In broad terms, Adaptive navigation support is about links and Adaptive presentation about content. 


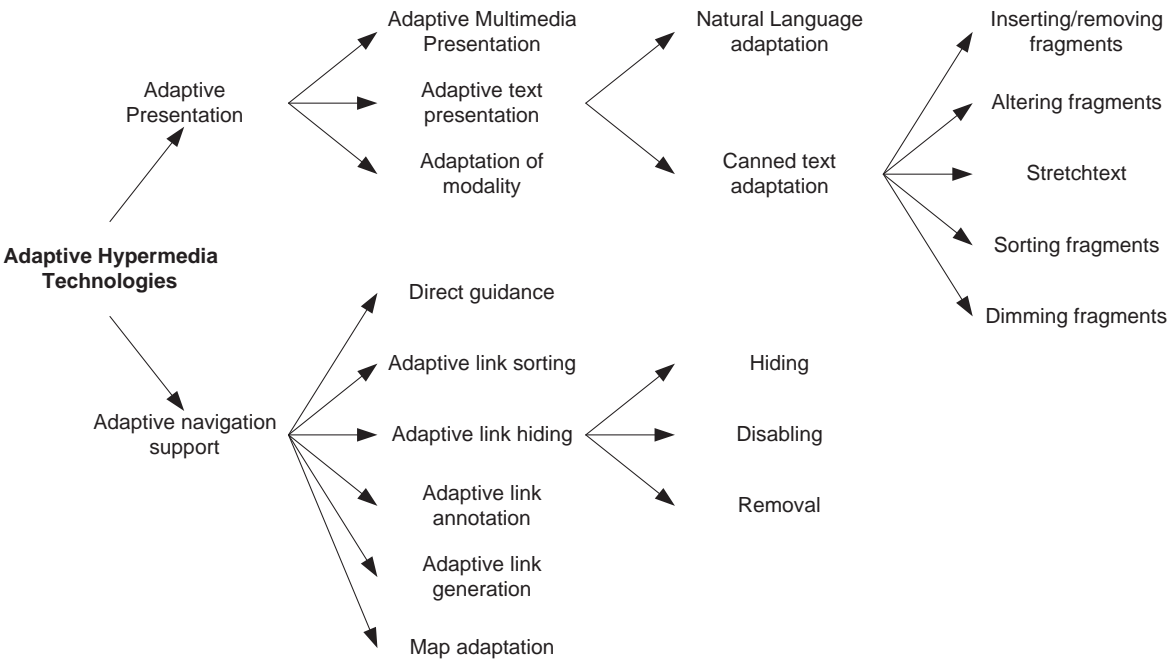

Fig. 1. Updated taxonomy of adaptive hypermedia technologies [4]

While the distinctions of the taxonomy are important for identification and classification of adaptive systems, the implementation of these techniques can be achieved using a small selection of fundamental data structures that can be combined to create powerful AH systems.

\section{Structures for Adaptive Hypermedia}

In this section we present a selection of hypermedia structures that could be used to represent the underlying information within an adaptive system. It is not our intention that these structures be exhaustive, more that they provide enough richness to support Brusilovsky's taxonomy. The structures are represented using the Fundamental Open Hypermedia Model (FOHM).

\subsection{Data}

The Data object (shown in Fig. 2) is the basic building block of FOHM, it can represent any piece of media (text, graphic, video stream, etc.). A Context object can be attached to the data and describes the context in which the data item is visible (the precise format of a context object is not specified in FOHM although one might imagine a set of key/value pairs as a basic format). When used in an AH system, the context of the data item would be matched against a user's profile to see whether the data item should be hidden from the user. 


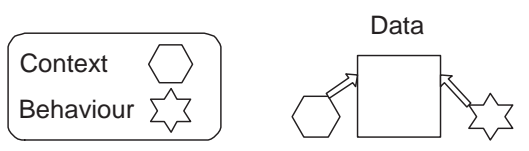

Fig. 2. FOHM Data structure

In addition to context, Behaviour objects can be attached to a Data object. These describe an action that occurs as a result of an event associated with the object they are attached to. In an $\mathrm{AH}$ system this can be used to modify the user profile, for example, in the case of data, the behaviour event might be 'on display' with an action of setting a flag in the user profile stating that the information in that data item has been read. Any technique from Brusilovsky's taxonomy that manipulates content might utilise data objects, while context and behaviour can be used for modelling prerequisite information in the system.

\subsection{Link}

Navigational link structures are represented in FOHM as Associations, bound to these are Reference objects that are used to address Data objects or other Associations. Fig. 3 shows a Link with three Data members, the first is bound as a source and the other two as destinations.

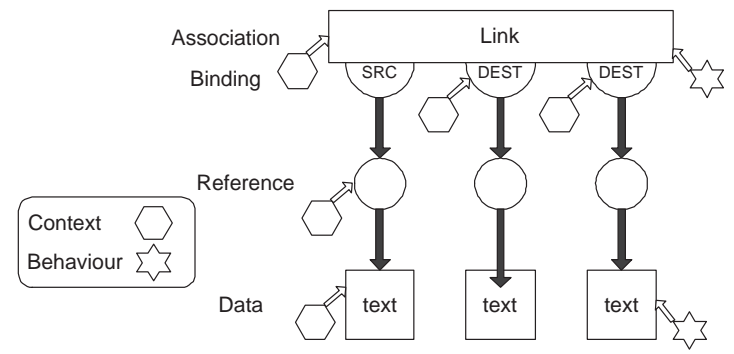

Fig. 3. FOHM Navigational Link

Context objects can be placed on Associations in a number of positions. When attached to the link, it can be used to scope the link (i.e. the link is only visible to the user if their profile matches the link's context). If the context is placed on the destination bindings instead, then while the link will always be visible, the destinations will change depending on the profile.

Behaviour objects can also be attached to Associations. For example, if we attach a behaviour with the event 'on traversal' then, when the user follows the link, the action specified in the behaviour object would be enacted by the system.

Contextual link structures could be used to implement any of the 'Adaptive Link Hiding' techniques described by Brusilovsky. For example a link could be authored with a context object attached that specified that it was only visible to an expert. When a novice queries the system for links, their user profile does not match against the link's context and the link is effectively hidden. 


\subsection{Tour}

A Tour is an association that represents a set of objects designed to be viewed in sequence. These objects might be data items representing a sequence of pages, association objects representing a sequence of sub structures or a mixture of both.

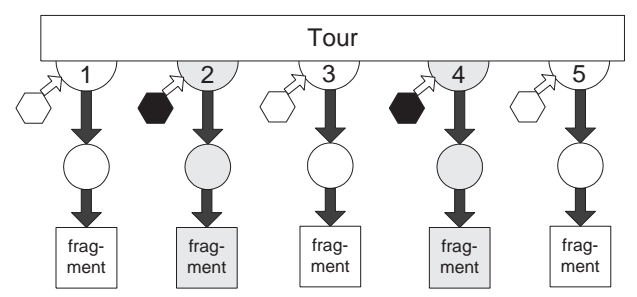

Fig. 4. Pruning the hyperstructure using context. The black contexts have failed to match leading to the pruning of the grey sub-structures.

A tour of data objects could be used to implement Brusilovsky's 'Inserting/Removing Fragments'. The client would query the link server to extract the structure shown in Fig. 4. Before being returned by the link server, it is pruned to remove sub-structures whose context does not match the user profile. The client inserts the remaining sub-structures, in the order they appear in the tour, into the document.

\subsection{Concept}

A Concept association can be used to collect together multiple objects that represent the same conceptual entity (for example the same piece of text in different languages). Brusilovsky's 'Adaptation of Modality' could be implemented with such a structure. Different media representations of the same information (e.g. video, audio, text) could be gathered together in a concept and then selected via querying with a particular user profile. When the concept structure is returned it will contain only those members that fit the query profile. The concept might be structured to be mutually exclusive, or to allow for multiple representations to be applicable at any given time.

\subsection{Level Of Detail (LoD)}

Like the concept, the LoD structure associates multiple representations of the same object. Unlike the concept structure, these representations are ordered within the association, from the simplest representation to the most complex. When queried in context the members of the LoD will be filtered. The application can then choose which of the remaining bindings to display according to what level of detail the current user would prefer.

For example, a system could support 'Stretchtext' from the taxonomy by storing a LoD structure containing data objects representing text at increasingly advanced 
levels. Initially the lowest positioned data object in the LoD would be displayed. When the user selects the text it could be replaced with the next available data object in the LoD.

\subsection{Equator "City" Example}

It is possible to combine these structures to produce more advanced effects (for example a tour of concepts). Before we go any further it is useful to see how they have been used in a real-world application.

City is a museum-based sub-project of the Equator Interdisciplinary Research Collaboration (IRC). The premise of the City project is that data objects represent real world locations so that adaptive structure can be authored about them. These structures can then be queried contextually to obtain personal views of the physical space (interpreted for the user via a device such as a PDA). Fig. 5 shows the structures that we are using.

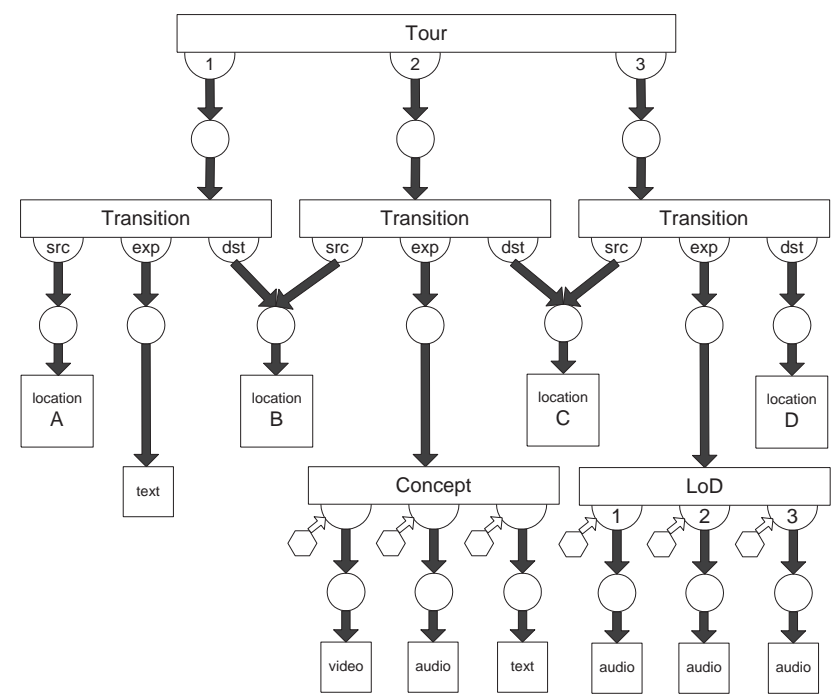

Fig. 5. FOHM Tour, Concept, LoD and Transition structures

The fundamental structure used in City is a Transition. This is very similar to a link in that it indicates a navigational move from one Data object $(s r c)$ to another $(d s t)$. Unlike a link it also includes an explanation (exp) member that explains why that move is relevant. For example, when moving from $\mathrm{A}$ to $\mathrm{B}$, the explanation might describe in what ways the objects at location $\mathrm{B}$ are related to those at location $\mathrm{A}$.

We have used the structures previously described along with these transitions to provide AH functionality. Curators of the museum space can organise sets of these transitions into tours that describe a number of steps that a visitor might like to take around the museum space. At any particular location the visitors' PDAs would 
present the available transitions that are available given the users current context, including any tours that progress from that point.

We can also use the concept and $L o D$ structures to tailor the explanations of transitions. For example on the $\mathrm{B}$ to $\mathrm{C}$ transition there are explanations available in several formats, while on the $\mathrm{C}$ to $\mathrm{D}$ transition there are several audio explanations of different lengths and complexity. When retrieving these structures, the users context will determine which explanation they will actually see.

\section{Revisiting the Taxonomy}

Having discussed the fundamental structures that can be used to provide adaptivity and seen how they can be used in a novel AH application, Fig. 6 illustrates how these structures could be used to implement the various adaptive techniques described in Brusilovsky's taxonomy.

The legend in Fig 6 is divided up between the two fundamental objects (data and links) and the extended FOHM structures. In addition, some of the techniques in the taxonomy require extra resources such as System Information (e.g. user access logs), or a Weight Metric that describes not only which parts of the structure match in a particular context but also how well they match.

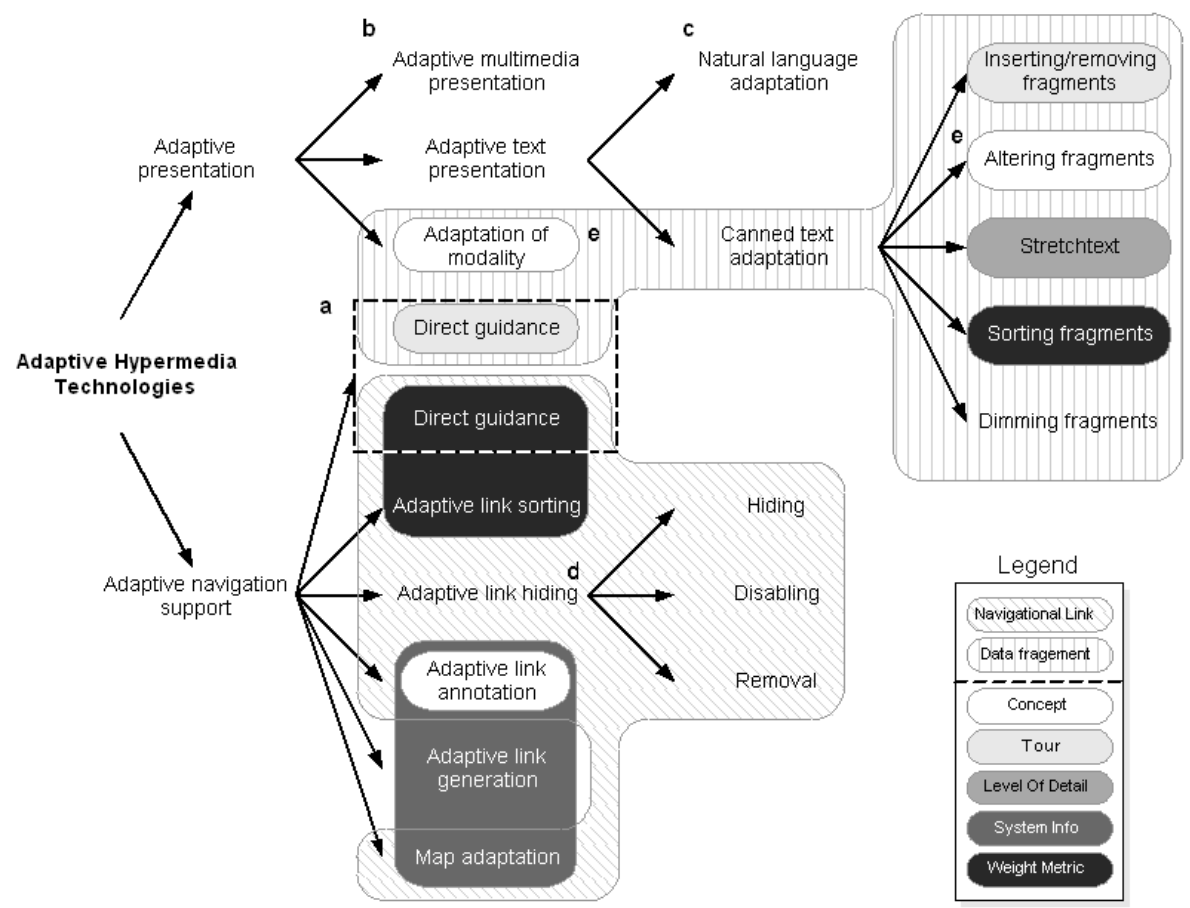

Fig. 6. A structural view of Brusilovsky's taxonomy that shows how FOHM structures can be combined to implement a range of adaptive hypermedia techniques. 
Each technique in the taxonomy is built up using the structures or resources required to implement that technique. For example, 'Adaptive link annotation' would require explicit link structures to store each hyperlink and collections of alternative annotations for each link would be stored in a concept structure. Finally the adaptive system would analyse the system information (in the form of user history) when choosing which annotation to present to the user.

The only visual change to the taxonomy that was required, involves duplicating 'Direct Guidance' (labelled $\boldsymbol{a}$ on Fig 6) as it was felt that this technique has two possible implementations.

The first approach could use a tour of the information domain; the adaptive 'next' button would therefore point to the next appropriate destination on the tour. An alternative approach would be to apply a link sorting routine (requiring a weight on each link) and then automatically select the link with the highest weight as the next destination.

One of the philosophies behind OHSs is that there is a general view of data that covers all media types. From this point of view, it can be seen that any of the 'Adaptive text presentation' techniques described in the taxonomy can also be applied equally well to 'Adaptive multimedia presentation'. To improve clarity, both techniques could be combined and re-labelled 'Adaptive Media Presentation'. This issue is highlighted as $\boldsymbol{b}$ in Fig. 6.

The same perspective leads us to question the way that Natural Language (NL) Adaptation ( $c$ in Fig. 6) is placed under text adaptation. Because the same techniques can be used with any media type, we believe that NL adaptation falls under a larger umbrella of 'Adaptive Sequencing', which might use canned or constructed information fragments. Moreover, NL adaptation influences nearly every single technique in the taxonomy. Whenever information fragments (or pages) are sequenced together, there is a need to conserve the progression of the narrative flow. In these situations, NL techniques, and wider sequencing methods in general, can be used as the glue to join fragments together.

Another consideration is that the various subcategories of 'Adaptive link hiding' (hiding, disabling and removal, labelled $\boldsymbol{d}$ ) are all structurally equivalent. This means that in a system that uses navigational link objects to implement a particular technology, for example link hiding, it has in place all the requirements needed to implement any of the other adaptive link techniques.

The diagram also shows the apparent similarity between 'Adaptation of modality' and 'Altering fragments' labelled $\boldsymbol{e}$ in Fig 6. These two techniques are functionally identical if one considers that fragments can contain multiple media representations of the same data objects. In such cases, choosing the best media type to display (adaptation of modality) is a process of selecting one fragment from a set of fragments (altering fragments).

\subsection{Reflections on Brusilovsky's Taxonomy}

Brusilovsky's taxonomy originally provided a mechanism for classifying the various AH systems at the time. Since then, more systems have been developed, some 
of which fit in to the existing taxonomy, and others that have forced extensions to the taxonomy making it more lengthy and complex.

With our focus on structure we propose that many techniques described by the taxonomy as textual are in fact applicable to a wide range of media.

It initially seems clear that the taxonomy divides structurally into two halves. The upper half is concerned with content, and requires a contextual data representation; the lower is concerned with navigation, and requires links with contextual membership. However, we have argued that Natural Language Adaptation impacts on both halves of the taxonomy and therefore blurs the division of the taxonomy into presentation and navigation. In our opinion both adaptive presentation and adaptive navigation are about adaptive sequencing. The first is concerned with intra-document adaptive sequencing, the second in inter-document adaptive sequencing. The difference concerns the presentation of the sequence i.e. as a linearisation or as a hyperstructure.

Other aspects of the taxonomy can also become blurred when viewed with a structural eye. In particular Map Adaptation seems a vague term that is concerned with the visualisation of many other aspects of the taxonomy (such as link hiding, altering fragments etc.) rather than being a category in its own right.

\subsection{Reflections on Auld Linky}

Auld Linky was designed as a contextual link server to deal with all the structures discussed in this paper. However, it is unable to totally support the taxonomy of techniques.

Linky is designed only to store and serve structure and for any particular problem clients need to be written that interpret the structures served by Linky and present them to the user. On its own Linky supports the first five criteria in the legend of Fig. 6 (the contextual structures). If a client were written that maintains a basic user history then it would also be able to support the sixth criteria, System Information. We feel that this division of responsibility is correct.

The reason Linky cannot support the entire taxonomy of techniques is because it lacks support for the seventh criteria, Weight Metrics. Currently Linky's matching process is binary (if a structural element does not match the users current context then it is culled from the context) and therefore provides no feedback on the quality of the match. The client is left unable to sort the resulting structure; a process needed to implement two of the techniques in the taxonomy, 'Adaptive Link Sorting' and 'Sorting Fragments'.

\section{Conclusions}

While both the $\mathrm{OH}$ and $\mathrm{AH}$ communities exist largely independently of each other, there are many areas where crossover could yield new research directions and offer solutions to shared problems. In this paper we have used Brusilovsky's taxonomy as a means to show how the structural $\mathrm{OH}$ approach could inform $\mathrm{AH}$ research, but also to 
explore what our own $\mathrm{OH}$ research, particularly the development of our contextual structure server, Auld Linky, could learn from established AH techniques.

$\mathrm{OH}$ 's emphasis on structure provides the means to implement a wide range of adaptive technologies and helps clarify the taxonomy. However it neither completely covers the taxonomy, nor provides the best programming solution in every situation. As we have shown, to implement every $\mathrm{AH}$ feature would require additional information such as system information and weight metric's.

We believe that $\mathrm{AH}$ systems that acknowledge the structural equivalence of many $\mathrm{AH}$ techniques have an advantage in that they may handle adaptation consistently across different techniques and media.

We also believe that any contextual $\mathrm{OH}$ server needs to provide for the problem domains being explored in $\mathrm{AH}$ research. To this end we are exploring the possibility of adding contextual weight metrics to the structures served by Auld Linky to allow it to support all the techniques described by Brusilovsky.

\section{Acknowledgements}

This research is funded in part by EPSRC Interdisciplinary Research Collaboration (IRC) project "EQUATOR" GR/N15986/01. Thanks to the Equator City Project, particularly Matthew Chalmers and Ian McColl of the University of Glasgow.

\section{References}

1. Anderson, K.M., Taylor, R.N. and Whitehead, E.J. (1994). "Chimera: Hypertext for Heterogeneous Software Environments". In ECHT '94. Proceedings of the ACM European conference on Hypermedia technology, Sept. 18-23, Edinburgh, Scotland, UK, pp 94-197, 1994.

2. Bailey C., El-Beltagy, S.R. and Hall, W. (2001). "Link Augmentation: A ContextBased Approach to Support Adaptive Hypermedia". In Proceedings of the 3rd Workshop on Adaptive Hypertext and Hypermedia, August 14-18, Århus, Denmark, pp 55-62, 2001.

3. Brusilovsky, P. (1996). "Methods and Techniques of Adaptive Hypermedia". Journal of User Modelling and User-Adaptive Interaction 6, n 2-3, pp 87-129.

4. Brusilovsky, P. (2001). "Adaptive hypermedia". User Modeling and User-Adapted Interaction, Ten Year Anniversary Issue 11 (Alfred Kobsa, ed.), pp 87-110.

5. Brusilovsky, P., Eklund, J. and Schwarz, E. (1998). "Web-based education for all: A tool for developing adaptive courseware". Computer Networks and ISDN Systems. Proceedings of 7th International World Wide Web Conference, April 1418, 30 (1-7), pp 291-300, 1998.

6. Chen, L. and Sycara, K. (1998). "WebMate: Personal Agent for Browsing and Searching". In Proceedings of the 2nd International Conference on Autonomous Agents, St. Paul, MN, May. ACM Press, New York, NY. pp. 132-139.

7. Davis, H.C., Rizk A. and Lewis A.J. (1996). "OHP: A Draft Proposal for a Standard Open Hypermedia Protocol". In Uffe Kock Wiil and Serge Demeyer, 
editors, Proceedings of the 2nd Workshop on Open Hypermedia Systems, ACM Hypertext '96, Washington, D.C., March 16-20, 1996.

8. De Bra, P. and Calvi, L. (1998). "AHA! An open Adaptive Hypermedia Architecture”. The New Review of Hypertext and Multimedia 4, 115-139.

9. Espinoza, F. and Höök, K. (1997). “A WWW Interface to an Adaptive Hypermedia System”. Presented at PAAM'96, London, UK, April 1996.

10.Fountain, A.M., Hall, W., Heath, I. and Davis, H.C. (1990). "MICROCOSM: An Open Model for Hypermedia With Dynamic Linking”. In A. Rizk, N. Streitz, and J. Andre, editors, Hypertext: Concepts, Systems and Applications (Proceedings of ECHT'90), pp 298-311. Cambridge University Press, 1990.

11.Grønbæk, K. and Trigg, R.H. (1994). "Design issues for a Dexter-based hypermedia system". Communications of the ACM, 37(3) pp 40-49, Feb. 1994.

12.Hall, W., Davis, H., Hutchings, G. (1996). "Rethinking Hypermedia: The Microcosm Approach" Electronic Publishing Series, No 4. Kluwer Academic Pub; ISBN: 0792396790.

13.Hothi, J. \& Hall, W. (1998). “An Evaluation of Adapted Hypermedia Techniques using Static User Modelling". In Proceedings of the 2nd Workshop on Adaptive Hypertext and Hypermedia, Pittsburgh, USA, June 20-24, pp 45-50, 1998.

14.Lieberman, H. (1995). "Letizia: An Agent That Assists Web Browsing". In Proceedings of the International Joint Conference on Artificial Intelligence, Montreal, August 1995.

15.Maglio, P.P. and Farrell, S. (2000). "LiveInfo: Adapting web experience by customization and annotation". In Proceedings of the 1st International Conference on Adaptive Hypermedia and Adaptive Web-based Systems. AH2000, Trento, Italy, August 2000.

16.Michaelides, D.T., Millard, D.E., Weal, M.J. and De Roure, D., C. (2001). “Auld Leaky: A Contextual Open Hypermedia Link Server". In Proceedings of the 7th Workshop on Open Hypermedia Systems, ACM Hypertext 2001 Conference. Aarhus, Denmark 2001.

17.Millard, D.E., Moreau, L, Davis, H.C. and Reich, S. (2000). "FOHM: A Fundamental Open Hypertext Model for Investigating Interoperability Between Hypertext Domains". In Proceedings of the '00 ACM Conference on Hypertext, May 30 - June 3, San Antonio, TX, pp 93-102, 2000.

18.Perkowitz, M. and Etzioni, O. (1999). "Towards adaptive web sites: Conceptual cluster mining". In Proceedings of the 17th International Joint Conference on Artificial Intelligence, 1999.

19.Stern, M.K. \& Woolf, B.P. (1998). "Curriculum Sequencing in a Web-Based Tutor”. In the Proceedings of Intelligent Tutoring Systems, 1998. 